\title{
Inequities in resources and preparedness for surgical complications of caesarean section in southern Gauteng hospitals
}

\author{
T S Maswime, ${ }^{1} \mathrm{MB} \mathrm{ChB}, \mathrm{FCOG}(\mathrm{SA})$, MMed; E J Buchmann, ${ }^{2}$ FCOG (SA), MSc, PhD \\ ${ }^{1}$ Wits Obstetrics and Gynaecology Clinical Research Division, University of the Witwatersrand, Johannesburg, South Africa \\ ${ }^{2}$ Obstetrics and Gynaecology Department, University of the Witwatersrand, Johannesburg, South Africa
}

Corresponding author: T S Maswime (smaswime@gmail.com)

Background. The number of maternal deaths from bleeding during and after caesarean section (BDACS) has increased in South Africa. Health-worker training and health-system strengthening are considered important prerequisites for improving maternal health outcomes. Objectives. To determine preparedness for, and health-system constraints to, safe caesarean section in southern Gauteng hospitals. Methods. This was a cross-sectional study in 15 hospitals. Data were collected by questionnaire from clinical heads of department in each hospital.

Results. The 15 hospitals included 5 district hospitals, 7 regional hospitals and 3 central (university academic) hospitals. The number of deliveries per hospital ranged from 893 to 44256 for 2013 - 2014, with a total of 201314 births and 70095 caesarean sections (34.8\%). Despite similar numbers of births, there were 20 deaths from BDACS at regional hospitals and 6 at central hospitals $(p=0.008)$. Service delivery constraints included an unequal staff distribution between central hospitals and lower levels of care, as well as non-availability of essential drugs and a lack of surgical capacity to arrest severe haemorrhage at district and regional hospitals.

Conclusion. The findings of this study reflect inequity in maternity services. Compared with central academic hospitals, district and regional hospitals are inadequately prepared for the management of complications from BDACS.

S Afr J Obstet Gynaecol 2016;22(1):21-24. DOI:10.7196/SAJOG.2016.v22i1.1039

The number of maternal deaths in South Africa (SA) from bleeding during and after caesarean section (BDACS) has increased in the last triennium (2011 - 2013), and over 70\% of the deaths were found to be clearly avoidable. ${ }^{[1]}$ A majority of maternal deaths in SA occur in regional and district hospitals. ${ }^{[2]}$ The National Committee on Confidential Enquiries into Maternal Deaths has recommended improvements in healthcare systems and healthcare workers' skills to improve the safety of maternity units and the ability to manage obstetric emergencies. ${ }^{[2]}$ For improving caesarean safety, a stepwise approach is suggested for the management of BDACS in the SA Saving Mothers: Caesarean Section Monograph, ${ }^{[3]}$ which includes the use of uterotonics, and surgical techniques to arrest haemorrhage.

Clinical audit is used for monitoring and reporting on health outcomes and process of care. Audit permits the review of adverse events, such as near-misses (women who survived life-threatening complications of pregnancy and childbirth) and maternal mortality, to review and improve quality of care. Clinical audits are based on a commitment to improve the healthcare system. ${ }^{[4]}$

A health system is made up of staff, funding, supplies, transport, communications, and leadership. ${ }^{[5]}$ There are various models for health-system frameworks. The World Health Organization (WHO)'s health-system framework has six key components which include governance, financing, medical technologies, health workforce, information and research, and service delivery ${ }^{[6]}$ Oliviera-Cruz et al. ${ }^{[7]}$ described health-system constraints to improving access to priority healthcare. The service delivery constraints include shortage and distribution of appropriately qualified staff, weak management and supervision, inadequate drugs and medical supplies, and lack of equipment and infrastructure.

Health-system strengthening is a prerequisite to achieving any improvement in health outcomes. It has been shown that focusing on disease priorities without improving health systems does not improve overall outcomes, ${ }^{[8]}$ hence the prioritisation of healthcare system improvements, as well as health-worker training, by the National Committee on Confidential Enquiries into Maternal Deaths. $^{[2]}$

The aim of this study was to evaluate preparedness for, and health system constraints to, provision of safe caesarean section in hospitals in southern Gauteng, and to link these to hospital levels of care.

\section{Methods}

A prospective cross-sectional multicentre study was conducted in southern Gauteng, in 15 public hospitals from July 2014 to May 2015. Gauteng Province consists of Johannesburg, Pretoria and numerous surrounding towns and cities, and has the highest number of deliveries in SA per year (annual facility live births in 2013, $n=208$ 710). ${ }^{[1]}$ Southern Gauteng is a metropolis with four districts, Ekurhuleni, Johannesburg, Sedibeng and West Rand. The districts are served by 18 government hospitals with maternity units, and with the exception of one of these (Tembisa Hospital) form part of the University of the Witwatersrand cluster of hospitals, including 3 central hospitals (all teaching hospitals in Johannesburg itself), 7 regional hospitals, and 7 district hospitals. All but 2 of the district hospitals, and all the regional hospitals, serve towns and adjacent high-density settlements to the east, west and south of Johannesburg. All hospitals receive high-risk patients who cannot be managed by clinics and midwife obstetric units. District hospital maternity units are staffed by midwives and medical officers, and may employ parttime specialists. They conduct normal and assisted deliveries, and caesarean sections, and refer patients they cannot manage to higherlevel hospitals. Regional hospitals offer specialist maternity care and referral services for district hospitals. They employ specialists, 
medical officers and interns, and generally have intensive care and high-care units on site. Certain patients, when a difficult birth is expected, may be referred to central hospitals. Central hospitals provide specialist obstetric care and subspecialty care. Their staffing is made up of interns, medical officers, registrars, specialists and subspecialists. Academic programmes for medical students, registrars and subspecialist trainees take place in the central hospitals. One of the central hospitals, officially considered to be a regional hospital by the Department of Health, is defined as a central hospital in this study, as it is an academic tertiary referral hospital with a large complement of specialists, and part of the University of the Witwatersrand's training platform for medical students and registrars.

\section{Ethical approval}

Ethical and institutional approval for the study was obtained from the University of the Witwatersrand's Human Research Ethics Committee, and from the Gauteng Department of Health. Individual hospital permission was received from the chief executive officers of the 17 University of the Witwatersrand cluster hospitals.

\section{Interviews - data collection}

In July and August 2014, the researcher conducted interviews with the clinical head of obstetrics in each hospital, using a structured questionnaire. The interviews set out to determine staffing, supervision and preparedness for surgical complications at caesarean section in southern Gauteng. The researcher asked questions about whether the hospitals were appropriately staffed for the number of deliveries conducted. The number of staff members was calculated according to the number of full-time staff members on the monthly current obstetric department timetable. Supervision was divided into teaching and monitoring. Teaching was assessed by determining the teaching methods in each hospital, and the number of caesarean sections done under supervision.

Monitoring was assessed according to whether maternal deaths, adverse events and maternal near-misses were discussed routinely in each hospital. Preparedness was assessed by the availability of drugs, facilities and surgical skills. Drugs were evaluated by availability in the hospital, and whether the drugs were being used according to the clinical head's knowledge. Facilities were evaluated by the availability of a blood bank, postoperative recovery area, obstetric high-care unit, and an intensive care unit (ICU) on site.

Skills were evaluated according to the competence of the doctors in surgical procedures at each hospital. The clinical heads were familiar with their doctors' skills levels and which surgical procedures were performed in each hospital. The interview inquired about the availability of staff that could perform life-saving procedures related to BDACS, such as obstetric hysterectomy and B-Lynch compression suture 24 hours a day on 7 days per week.

Data analysis employed quantitative techniques. Maternal mortality ratio was calculated per hospital as the institutional maternal mortality ratio (iMMR). In the absence of live birth data, the denominator used for the iMMR was a live birth approximation of 0.98 times the total births, assuming a general SA stillbirth rate of $2 \%$. Descriptive data were analysed using medians and ranges for continuous variables, and proportions with percentages or rates for categorical variables. Comparisons of categorical data were made using the $\chi^{2}$ test, and pairwise correlation was done using scatter plots with Spearman's rank correlation for non-parametric data. Statistical significance was indicated by $p<0.05$.

\section{Results}

The study was conducted in 15 hospitals: 5 district hospitals, 7 regional hospitals and 3 central hospitals. Permission was sought from 17 hospitals, but 2 district hospital managers declined participation. In 2 hospitals the interviews were conducted with a specialist appointed by the clinical head. Four clinical heads asked to complete the questionnaire themselves, and return it to the researcher. In 11 of the hospitals the researcher was referred to the midwife in charge of the labour ward for questions about the nursing staff. The questionnaires were completed on the day of the interview. The researcher collected maternity statistics for 2013 and 2014 from the midwife in charge of the labour ward in 7 hospitals, from data capturers in 5 hospitals, and from the clinical heads in 3 hospitals. The statistics for 2014 were collected from February to April 2015.

In the 15 hospitals there were 201314 births in 2013 and 2014, with 70095 caesarean sections (34.8\%). The total number of births at each of the hospitals

Table 1. Full-time staffing and obstetric outcomes in $\mathbf{1 5}$ hospitals in southern Gauteng, 2013 - 2014, by levels of care

\begin{tabular}{|c|c|c|c|}
\hline & District $(N=5)$ & Regional $(N=7)$ & Central $(N=3)$ \\
\hline \multicolumn{4}{|l|}{ Aggregated data per level of care } \\
\hline Total births, $n$ & 25254 & 89243 & 86678 \\
\hline Maternal deaths, $n$ & 10 & 186 & 114 \\
\hline iMMR per 100000 live births & 40 & 213 & 134 \\
\hline Caesarean sections, $n$ & 5919 & 30833 & 33343 \\
\hline Hospital caesarean section rate, $\%$ & 23.4 & 34.5 & 38.5 \\
\hline Maternal deaths from BDACS, $n$ & 0 & 20 & 6 \\
\hline Obstetric specialists, $n$ & 1 & 12 & 51 \\
\hline All doctors, $n^{*}$ & 16 & 105 & 188 \\
\hline Doctors, $n / 10000$ births per annum & $9.9^{\dagger}$ & 23.5 & 43.4 \\
\hline Specialists, $n / 10000$ births per annum & $0.0^{\dagger}$ & 2.7 & 11.8 \\
\hline \multicolumn{4}{|l|}{ Hospital data, median (range) } \\
\hline Midwives & $18(12-29)$ & $28(12-39)$ & $55(44-204)$ \\
\hline Medical interns & $0(0-0)$ & $5(2-7)$ & $21(17-25)$ \\
\hline Medical officers & $3(2-4)$ & $10(3-10)$ & $5(2-10)$ \\
\hline Registrars & $0(0-0)$ & $0(0-2)$ & $17(13-27)$ \\
\hline Obstetric specialists & $0(0-1)$ & $1(1-4)$ & $12(11-28)$ \\
\hline All doctors & $3(2-5)$ & $16(8-20)$ & $52(46-90)$ \\
\hline Hospital caesarean section rate & $25.6(16.2-36.2)$ & $36.6(25.3-42.4)$ & $37.0(33.3-48.5)$ \\
\hline iMMR per 100000 live births & $43(0-62)$ & $204(77-350)$ & $136(47-240)$ \\
\hline
\end{tabular}


ranged from 893 to 44256 , with a median of 9063 . Caesarean section rates ranged from $16.2 \%$ at a district hospital to $48.5 \%$ at a central hospital (Table 1). The overall maternal mortality ratio was highest at regional hospitals (213 per 100000 live births) (Table 1). While the number of total births at regional and central hospitals was similar, BDACS accounted for 20 maternal deaths at regional hospitals and 6 at central hospitals $(p=0.008)$. The number of obstetric doctors per 10000 births was 9.9 at district hospitals, 23.7 at regional hospitals and 43.4 at central hospitals. The corresponding numbers for obstetric specialists were 2.7 and 11.8 for regional and central hospitals, respectively. However, the proportion of obstetric doctors per 10000 births at the 15 hospitals did not correlate significantly with the hospital maternal mortality ratios ( $p=0.68$ ) (Fig. 1).

Ten hospitals (66.7\%) trained interns, and 4 hospitals $(26.7 \%)$ trained registrars. Eleven hospitals (73.3\%) had at least one full-time specialist (range 1 - 28). The total number of doctors ranged from 2 in a district hospital with 4654 births to 97 in the tertiary hospital that had 44256 deliveries in 2013 and 2014.

The skill of teaching caesarean section technique was reserved for the most senior doctors in each hospital. All hospitals taught by observation and supervision, 6 hospitals (40.0\%) also used videos, and none used models. Three hospitals (20.0\%) stipulated a minimum number of caesarean sections that a new doctor had to perform under supervision prior to operating independently. All the hospitals held regular perinatal review meetings, at which maternal deaths were discussed. The 3 central hospitals, as well as 2 regional hospitals, held weekly meetings, and the remaining hospitals held monthly meetings. Nine hospitals $(60.0 \%)$ reported that they also presented maternal adverse events in their meetings. Three hospitals (20.0\%) discussed all maternal near-miss cases from BDACS at the meetings. Collaboration between hospitals, beyond referral, was limited to occasional attendance by regional and district hospital specialists at central hospital perinatal review meetings. There were no outreach programmes (e.g. specialist support visits) in place from higher levels to lower levels of care.

Oxytocin and misoprostol were available in all 15 hospitals. Ergometrine was only available in 10 hospitals (66.7\%) and

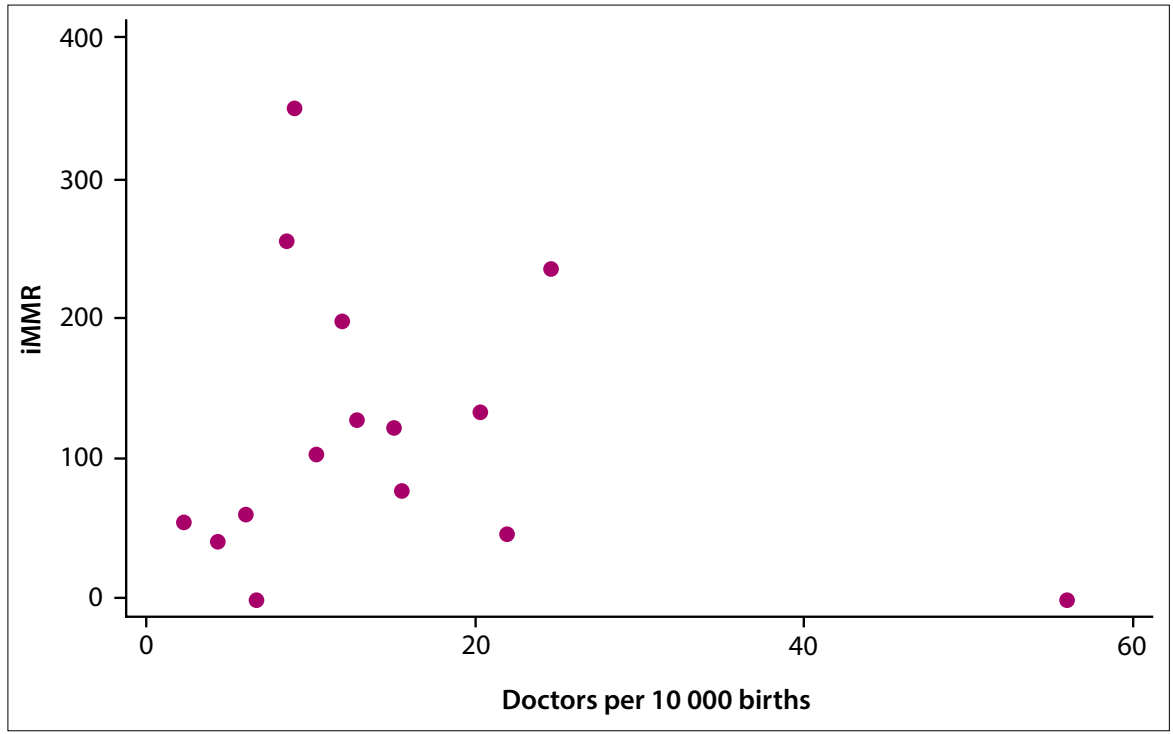

Fig. 1. Scatter plot showing institutional maternal mortality ratio per 100000 live births (iMMR) for 15 southern Gauteng hospitals correlated to numbers of doctors as a proportion of births at each hospital (Spearman's correlation coefficient $\mathrm{r}=0.12, \mathrm{p}=0.68$ ).

Table 2. Preparedness for life-threatening surgical complications of caesarean section in hospitals in southern Gauteng

\begin{tabular}{llll}
\hline & District $(\boldsymbol{N}=\mathbf{5}), \boldsymbol{n}(\%)$ & Regional $(\boldsymbol{N}=\mathbf{7}), \boldsymbol{n}(\%)$ & Central $(\boldsymbol{N}=\mathbf{3}), \boldsymbol{n}(\%)$ \\
\hline Available drugs & & & \\
Oxytocin and misoprostol & $5(100)$ & $7(100)$ & $3(100)$ \\
Ergometrine & $4(80)$ & $4(57.1)$ & $3(100)$ \\
Tranexamic acid injection & $3(60)$ & $7(100)$ & $3(100)$ \\
Prostaglandin F2-alpha & $1(20)$ & $1(14.3)$ & $3(100)$ \\
Ability to perform procedures & & & \\
B-Lynch compression suture & $3(60)$ & $3(42.8)$ & $3(100)$ \\
Obstetric hysterectomy & $3(60)$ & $7(100)$ & $3(100)$ \\
On-site facilities & & & $3(100)$ \\
Blood bank & 0 & $6(85.7)$ & $3(100)$ \\
High-care unit & $1(20)$ & $7(100)$ & $3(100)$ \\
24-hour recovery & $2(40)$ & $5(71.4)$ & $3(100)$ \\
24-hour theatre & $4(80)$ & $6(85.7)$ &
\end{tabular}

prostagladin F2-alpha was available in 5 hospitals (33.3\%). Tranexamic acid injection was available in 13 hospitals (86.7\%); however, only 2 of these hospitals (15.4\%) reported ever using it for obstetric patients. The central hospitals had all the uterotonic drugs available. Prostaglandin F2-alpha was only available in 1 district hospital, and 1 regional hospital. Only 4 of the 7 regional hospitals (57.1\%) had ergometrine in stock (Table 2).

All the hospitals performed caesarean sections; however, 2 (13.3\%) did not operate on all days of the week because of staffing shortages. On-site blood banks functioned in 9 hospitals (60.0\%), and the rest, including all the district hospitals, relied on emergency blood kept in refrigerators on site. One district hospital had an obstetric high-care unit. One central hospital did not have an ICU on site (Table 2).

Emergency skills to arrest obstetric haemorrhage were evaluated according to whether the staff were available on a 24-hour basis to perform these procedures in each hospital. Uterine balloon tamponade (Bakri balloon or condom balloons) had never been done in 12 hospitals (80.0\%), and B-Lynch compression sutures had never been done in 6 hospitals $(40.0 \%)$. None of the informants could recall ever performing a uterine tourniquet procedure using a Foley catheter at their hospitals. In two of the hospitals (13.3\%), the informants stated that 
none of the doctors was skilled to perform emergency hysterectomy during and after caesarean section (Table 2).

\section{Discussion}

A clear message has emerged of inequities in Gauteng maternity services. These inequities, mostly between regional hospitals and teaching centres, are unfortunate, given the relative sophistication of Gauteng Province in SA, with its medical schools and high-level central hospitals. As in the remainder of the country, the majority of maternal deaths occurred in district and regional hospitals, where medical staffing and facilities are most lacking. The relationship between overall maternal mortality and staffing levels per hospital is complex, and did not show any correlation, but there was a significant difference in numbers of maternal deaths related to BDACS between regional and central hospitals, with similar caesarean section rates. Observing overall maternal mortality ratios alone may therefore mask preventable maternal mortality, as is the case with BDACS. While the ideal is that there should be no deaths at all from BDACS, one would expect more deaths, not fewer, in central hospitals, given that high-risk obstetric patients, both preoperatively and postoperatively, should be cared for at high-level hospitals. The high maternal mortality in regional hospitals is therefore of concern. The inequities were greatest in medical staffing, and most obvious with specialist obstetricians. The central hospitals had twice as many doctors and more than four times more specialists per delivery than regional hospitals. It should of course be accepted that central teaching hospitals need more specialists than regional hospitals, but the difference here still amounts to a serious maldistribution of skilled human resources. In that context, the absence of any formal outreach activities from the central hospitals seems regrettable.

The inequities are further reflected in the relative lack of preparedness of regional and district hospitals for dealing with severe BDACS. Essential second-line drugs, such as tranexamic acid and prostaglandin F2-alpha, and especially ergometrine, were not available in several institutions, remarkably in three of the regional hospitals where difficult caesarean sections might be done. The inability to perform B-Lynch compression sutures at several regional hospitals was also notable. These deficiencies were not investigated in this study, but root cause analysis might find management failures, overburdened specialists and the lack of central hospital outreach activities to be significant culprits.

What was somewhat encouraging was the ability of all hospitals to provide emergency blood for transfusion. Also, all hospitals held perinatal review meetings, where maternal deaths were also discussed. Both of these successes may have resulted from implementing guidelines suggested in the Saving Mothers reports of the National Committee for Confidential Enquiries into Maternal Deaths. ${ }^{[2]}$

Inequities in emergency obstetric care are a fact across the world, most notably between rich and poor countries, ${ }^{[0]}$ but also within countries. Differences between rural and urban obstetric services ${ }^{[10,11]}$ are well described, as are the extreme differences between private and public healthcare overall in SA. ${ }^{[12]}$ However, there appear to be no reports of inequities in public obstetric services within a city region, such as southern Gauteng. Possible drivers of the inequity include university advocacy for optimal care in its training hospitals, unattractiveness to doctors of regional hospitals in economically depressed towns away from Johannesburg, budgetary constraints in the provincial health service, poor co-ordination of hospital referral systems, and management weakness at lower levels of care. Further research should investigate these and other possibilities relating to caesarean section preparedness, as well as emergency obstetric care as a whole. This study was limited by its focus on caesarean section safety in terms of resource limitations and outcomes, without consideration of upstream determinants of these deficiencies. Another limitation relates to the once-off cross-sectional nature of the evaluation, which cannot assess trends in resource capacity at the hospitals. However, the data stand as facts that suggest inequitable resource allocation for emergency obstetrics, which may be a problem in other SA metropolitan areas that have medical schools.

Several immediate interventions should be considered to improve caesarean section safety at the hospitals in southern Gauteng. Most of these relate to recommendations in the national Confidential Enquiries into Maternal Deaths report from 2008 to 2010:[2] (i) human resources needs and norms must be investigated, perhaps using the Workforce Indicators of Staffing Need (WISN) approach introduced to SA in $2012,{ }^{[13]}$ to create and fill obstetric specialist and medical officer posts in the regional and district hospitals; (ii) all maternal deaths and cases of near-miss related to caesarean section bleeding should be audited and discussed at regular perinatal review meetings; (iii) training in caesarean section technique needs to be formalised at district and regional hospitals, including using training videos, with a minimum of 10 caesarean sections done under supervision before 'solo' caesarean sections can be attempted; ${ }^{[14]}(i v)$ all midwives and obstetric doctors in the hospitals must be exposed to ESMOE (Essential Steps in Managing Obstetric Emergencies) training, which includes modules on surgical skills and obstetric haemorrhage; ${ }^{[15]}$ and $(v)$ sustainable emergency obstetrics outreach programmes from the central to the regional and district hospitals must be implemented and sustained, with university and provincial government support.

Acknowledgements. The research was supported by the Carnegie Corporation of New York (Grant B8749.R01), and the SA Medical Research Council Self-initiated Research Grant. The views and opinions expressed are not those of the funders, but of the authors.

\section{References}

1. Gebhart G, Fawcus S, Moodley FZ. Maternal death and caesarean section in South Africa: Results from the 2011 - 2013 Saving Mothers Report of the National Committee for Confidential Enquiries into Maternal Deaths. S Afr Med J 2015:105(4);287-291. DOI:10.7196.SAMJ.9351

2. National Department of Health. Saving Mothers 2008 - 2010: Fifth report on the Confidential Enquiries into Maternal Deaths in South Africa - Comprehensive Report. 2012. Pretoria: NDoH, 2012. www.doh.gov.za/list.php?type=Reports (accessed 19 June 2015).

3. Moodley J, ed. Saving Mothers: Caesarean Section Monograph. Pretoria: National Department of Health, 2013.

4. Graham W. Criterion-based clinical audit in obstetric care: Bridging the quality gap. Best Pract Res Clin Obstet Gynaecol 2009;23(3):375-388. DOI:10.1016/j.bpobgyn.2009.01.017

5. World Health Organization. Monitoring the Building Blocks of Health Systems: A Handbook of Indicators and their Management Strategies. Geneva: WHO, 2010.

6. World Health Organization. Key Components of a Well-Functioning Health System. Geneva: WHO, 2010.

7. Oliviera-Cruz V, Hanson K, Mills A. Approaches to overcoming constraints to effective health service delivery: A review of the evidence. J Int Dev 2003;15(1):41-65.

8. World Health Organization. Strengthening Health Systems to Improve Health Outcomes: WHO's Framework for Action. Geneva: WHO, 2007.

9. Pattinson R. Safety versus accessibility in maternal and perinatal care. S Afr Med J 2015;105(4):261265. DOI:10.7196.SAMJ.9182

10. Echoka E, Dubourg D, Makokha A, et al. Using the unmet obstetric needs indicator to map inequities in life-saving interventions at the local health care system in Kenya. Int J Equity Health 2014;13:112.

11. Stuckler D, Basu S, McKee M. Health care capacity and allocations among South Africa's provinces - inequality traps after the end of apartheid. Am J Public Health 2011;101:165-172. DOI:10.2105/ AJPH.2009.184895

12. Mayosi B, Lawn J, van Niekerk A, Bradshaw D, Abdool Karim S, Coovadia H. Lancet South Africa Team. Health in South Africa: Changes and challenges since 2009. Lancet 2012;380(9858):20292043. DOI:10.1016/S0140-6736(12)61814-5

13. World Health Organization. Workload Indicators of Staffing Need (WISN). A Manual for Implementation. Geneva: WHO, 1998.

14. Madsen K, Grónbeck L, Rifbjerg Larsen C, et al. Educational strategies in performing cesarean section. Acta Obstet Gynecol Scand 2013;92(3):256-263. DOI:10.1111/aogs.12055

15. Moran N, Naidoo M, Moodley J. Reducing maternal mortality on a countrywide scale: The role of emergency obstetric training. Best Pract Res Obstet Gynaecol 2015;29(8):1102-1118. DOI:10.1016/j.bpobgyn.2015.08.002 